\title{
Les études d'ondes de submersion des grands barrages d'EDF
}

\author{
G. Benoist EDF - Laboratoire national d'hydraulique, Chatou
}

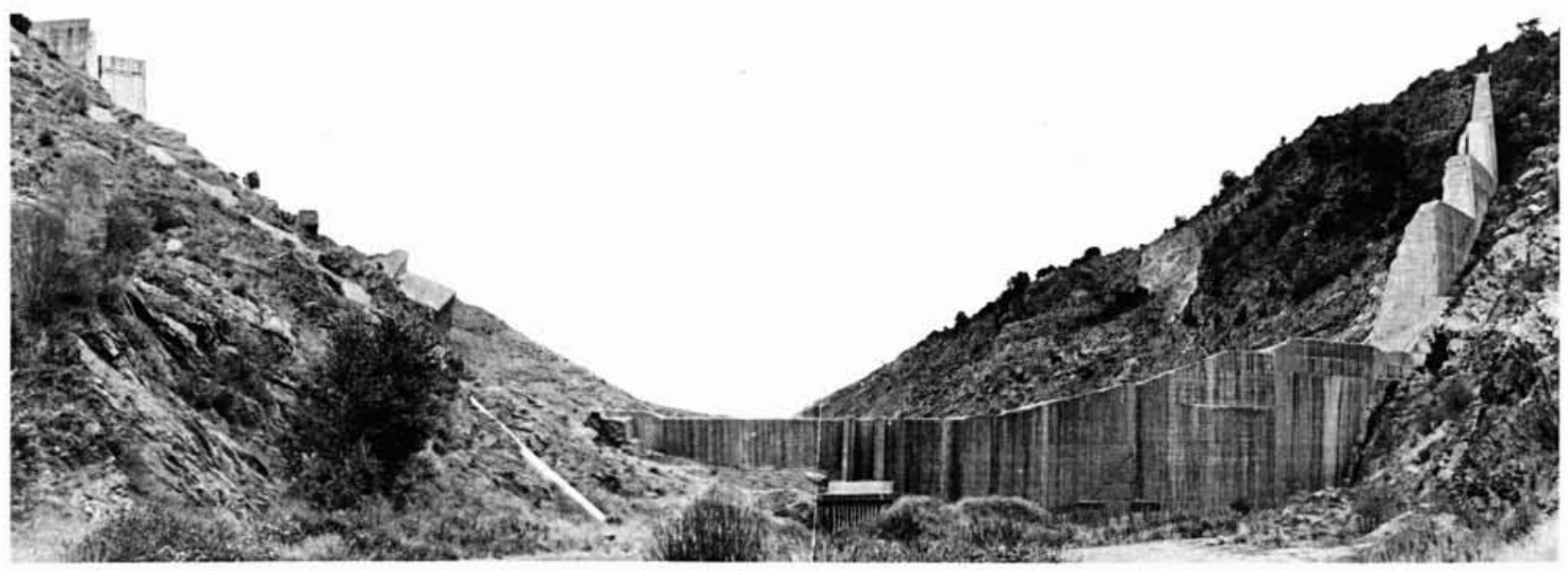

1. Le barrage de Malpasset après sa rupture, vu d'amont.

1. Rappel de la réglementation française en matière de sécurité des barrages

La réglementation française en matière de sécurité des barrages a été revue et complétée à la suite de la catastrophe provoquée par la rupture du barrage de Malpasset en 1959 (fig. 1).

En premier lieu a été créé, par décret du 13 juin 1966, le Comité technique permanent des barrages, organisme central et souverain qui est obligatoirement appelé à donner son avis sur les projets d'exécution de barrages de plus de $20 \mathrm{~m}$ de hauteur.
En second lieu, la réglementation relative à l'inspection et à la surveillance des barrages, qui datait de 1927 , a été rénovée par circulaire interministérielle du 14 août 1970. Cette circulaire attire notamment l'attention sur la surveillance particulière obligatoire lors de la première mise en eau et sur l'emploi d'appareils d'auscultation.

Entre temps, pour prévenir ou pallier les conséquences de la rupture d'un barrage, il a été décidé par décret du 16 mai 1968 de prendre des mesures destinées à organiser l'alerte et la protection des populations à l'aval des ouvrages les plus importants. Etaient ainsi rendus obligatoires l'établissement d'un plan d'alerte aux populations et l'installation de dispositifs d'alerte pour tous les

\section{The failure waves studies devoted to large dams at EDF}

The decree of may 1968 made compulsory to provide emergency plans for all the large french dams. Since that time, EDF has developed a lot of means of study of dam-break waves. The main tool is constituted by a computer code, based on the one-dimensional unsteady flow theory, and devoted to the full description of the floods. The particular features of the method are detailed, and also is the modelisation of the geometry of valleys. A view upon the experiments made to assess the method is given. Then, two auxiliary approaches are described, i.e. the scale-model tests and the two-dimensional model. Finally, a map of flooded areas is presented as an example of the results which are delivered in current study reports, and which constitute the main basis to establish the emergency plans. 

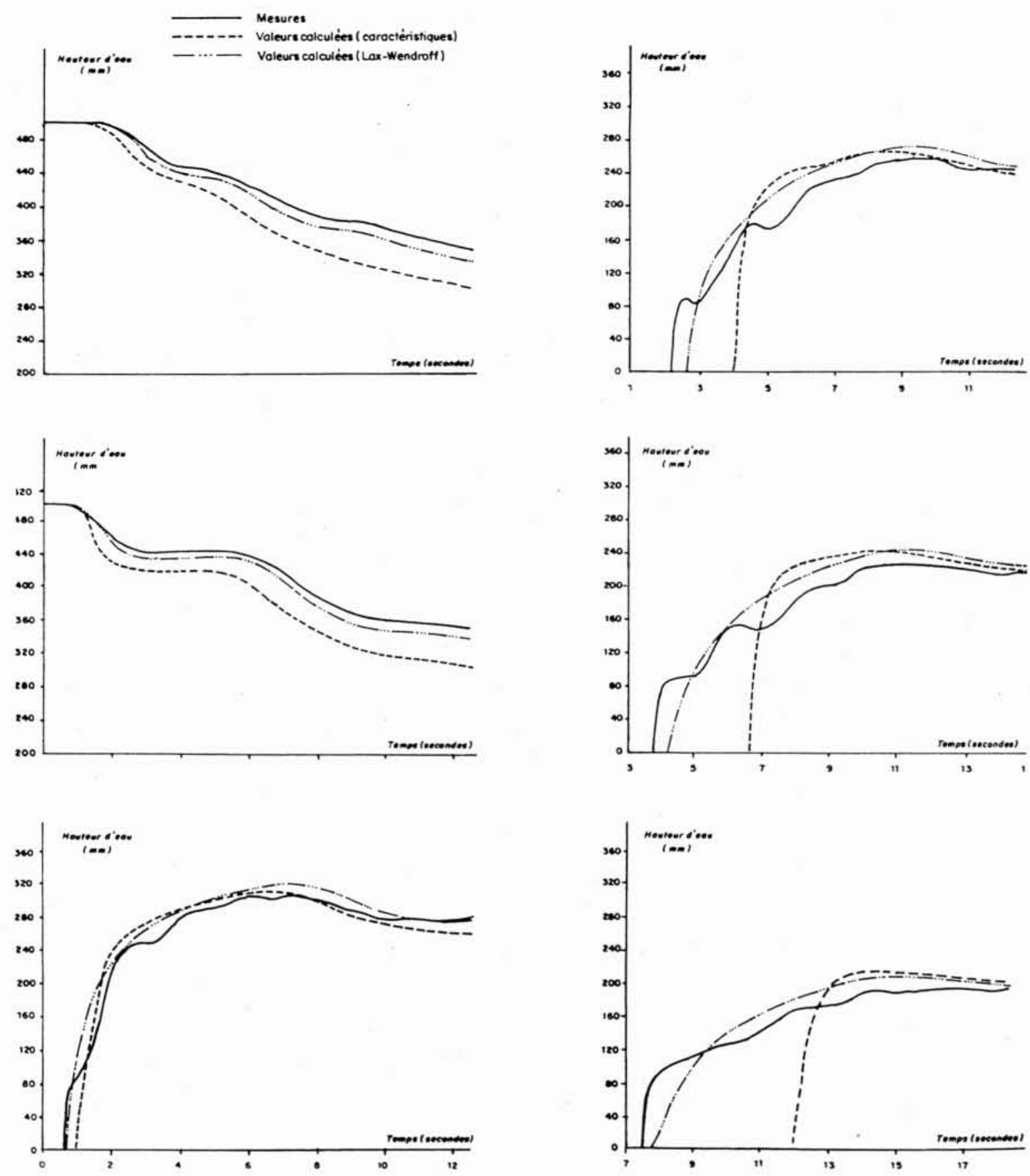

2. Confrontation des hauteurs d'eau calculées et mesurées sur le canal expérimental en différents points du modèle.

ouvrages de plus de $20 \mathrm{~m}$ de hauteur ayant une retenue d'au moins $15 \mathrm{hm}^{3}$ de capacité. Ces mesures seront d'autre part probablement étendues dans un proche avenir à des ouvrages de moindre importance.

Toute cette réglementation, instaurée pour faciliter la protection des populations à l'aval des barrages, repose sur le postulat qu'un barrage en exploitation ne se rompt pas brutalement sans signes précurseurs.

Après étude des statistiques mondiales portant sur une population de 10000 grands barrages, le législateur a estimé qu'il était préférable de ne mettre en état de vigilance particulière tous les responsables de la sécurité des barrages et des populations (exploitant, services de contrôle de l'Etat, autorités civiles, service de la protection civile) que dans certaines circonstances exception- nelles de la vie d'un barrage : première mise en eau, crue exceptionnelle, et constatation, grâce à la surveillance et à l'auscultation, de faits anormaux susceptibles de compromettre la sécurité.

La restriction à ces cas exceptionnels de l'état de vigilance renforcée est un gage d'efficacité de la protection : s'il est possible à l'être humain d'être particulièrement attentif à un événement pendant une courte période, il ne saurait rester à l'affût durant toute une vie. $\mathrm{Si}$ cette réglementation avait existé à l'époque de la catastrophe de Malpasset, elle aurait eu sa pleine efficacité. En effet, d'une part le barrage s'est rompu lors de son premier remplissage, d'autre part des mesures topographiques faites avant la rupture, mais dépouillées seulement après l'accident, ont montré que pour un 
remplissage partiel de la retenue, le pied de l'ouvrage s'était déplacé vers l'aval de 15 à $20 \mathrm{~mm}$.

Les enseignements tirés de cette catastrophe ont permis de réaliser de grands progrès en matière de conception, de surveillance et de sécurité des barrages. Un contrôle attentif de l'ouvrage, de ses fondations et des versants de la retenue permet de déceler les comportements anormaux, de prendre des mesures appropriées - vidange de la retenue par exemple - ou même de donner l'alerte si tout risque ne peut être écarté.

L'évacuation préventive des populations menacées par une rupture éventuelle est alors la seule mesure qui puisse être réellement efficace, et qui en particulier puisse permettre de sauver les habitants des régions atteintes en moins d'une heure par le flot. Les événements survenus en Californie à Baldwin Hills en 1963 et à San Fernando en 1971 confirment l'efficacité de mesures de cet ordre: dans les deux cas, les autorités, prévenues de la rupture imminente du barrage, ont pu organiser à temps l'évacuation de centaines de milliers de personnes.

\section{La naissance de l'outil d'étude d'EDF}

En tant que concessionnaire de la majorité des grands barrages français, EDF était concernée au premier chef par la nouvelle réglementation. En particulier, l'établissement des plans d'alerte exigeait de mettre au point des moyens d'étude des ondes de submersion consécutives à la rupture des barrages. Cette tâche échut tout naturellement au LNH, où dès 1960 des simulations de la rupture du barrage de Malpasset avaient été entreprises sur modèles réduits. Autour des années 70 , une véritable mobilisation des moyens disponibles - tant expérimentaux que mathématiques - fut engagée, centrée sur une équipe de recherche intégralement axée sur cette mise en œuvre. A l'issue de cette phase d'investigations se sont dégagés les principaux traits du profil de l'outil le mieux adapté au problème. Les choix essentiels relèvent d'abord de quelques considérations simples qui se résument comme suit :

A/ La longueur des vallées à étudier allant de quelques dizaines à plusieurs centaines de kilomètres, il était impensable que les études correspondantes soient menées sur des modèles physiques, dont les dimensions auraient été démesurées. Cela explique que l'effort principal ait dès l'abord été orienté vers la mise au point d'un code de calcul opérationnel, alors même que les capacités des ordinateurs et le développement des modèles numériques étaient incomparablement plus modestes qu'aujourd'hui.

B/ Le principal but recherché pour l'établissement des plans d'alerte était la délimitation des zones inondables en cas de rupture d'un barrage. Cela impliquait l'estimation la plus précise possible des niveaux d'eau atteints par l'onde de submersion le long d'une vallée. Pour cela une restitution fidèle de la topographie a paru indispensable. Cette option valait tout autant pour la modélisation numérique des vallées que pour l'outil mathématique de simulation de l'onde, qui devait être capable d'accepter tous les accidents de relief naturels sans risque d'incident numérique et sans altération de l'onde.

C/ Les hauteurs des barrages concernés par le programme d'étude se situent entre $20 \mathrm{~m}$ et $180 \mathrm{~m}$, la hauteur moyenne étant de $67 \mathrm{~m}$. Les capacités de leurs retenues s'étagent entre $15 \mathrm{hm}^{3}$ et $1270 \mathrm{hm}^{3}$, pour un volume moyen de $117 \mathrm{hm}^{3}$. Ces chiffres laissaient présager des hauteurs d'onde et des vitesses d'écoulement sans commune mesure avec les crues naturelles. Cette constatation entraînait plusieurs conséquences importantes : en premier lieu, il était illusoire de chercher à étalonner en rugosités les vallées à modéliser, en se basant sur des crues naturelles, et avec la technique habituelle de discrimination entre les écoulements en lits mineur et majeur. De plus, on pouvait escompter voir apparaître des régimes rapidement variables dans certaines zones d'écoulement, avec alternance de zones fluviales et torrentielles séparées éventuellement par des ressauts hydrauliques. On se devait donc de chercher à reproduire ces singularités des écoulements.

D/ La plupart des vallées françaises sur lesquelles sont implantés des barrages importants comportent schématiquement une zone de relief, étroite, de pentes assez raides et avec des parois plutôt escarpées, suivie à l'aval de zones de plaine avec un champ d'inondation et des pentes douces. On pouvait donc tabler dans la plupart des cas sur la prédominance du caractère filaire de l'écoulement de l'onde. Cela a permis de s'orienter vers un code de calcul monodimensionnel, qui aurait toute chance d'être applicable dans la majeure partie des études.

Ainsi se trouvait défini l'outil d'étude dans ses grandes lignes :

Il serait fait usage de modèles numériques, faisant appel à un code de calcul monodimensionnel d'écoulements non permanents, basé sur les équations de SaintVenant.

Le schéma de résolution numérique serait stable en présence des vallées naturelles, tout en assurant la conservativité des volumes. Les singularités d'écoulement pouvant entraîner des surélévations locales seraient prises en compte. La modélisation de la vallée serait fidèle, l'écoulement s'effectuant sur un lit global affecté de rugosités expérimentales.

Les choix définitifs ont été étayés par plusieurs séries d'essais en canaux expérimentaux ([1] et [2]). Ces essais ont permis d'étudier successivement :

- la propagation sur fond sec en canal uniforme ;

- la propagation sur un tirant d'eau initial en canal uniforme ;

- la propagation sur fond sec en présence d'un élargissement, d'un rétrécissement, puis des deux à la suite, - la propagation sur fond $\mathrm{sec}$ avec pénétration dans une retenue, propagation sur celle-ci, formation d'un ressaut et déversement sur un ouvrage.

Les canaux étaient dotés successivement de deux rugosités différentes. Les résultats d'essais ont été confrontés à ceux obtenus sur modèles mathématiques (fig. 2). 
En outre, des simulations de la rupture du barrage de Malpasset étaient effectuées sur un modèle non distordu, un modèle distordu, et un modèle mathématique [3].

La qualité des méthodes numériques mises à l'essai put ainsi être évaluée de manière intrinsèque et par comparaison aux résultats expérimentaux.

Sur le plan intrinsèque, les deux critères retenus furent la conservativité des volumes et l'absence d'oscillations d'origine numérique. Les tests comparatifs ont porté sur la méthode des caractéristiques locales et sur la méthode de Lax-Wendroff, dont on trouvera en [4] une description succincte. Ils ont permis de conclure en faveur de la méthode de Lax-Wendroff après certains aménagements de celle-ci destinés à éliminer ses défauts inhérents.

Ainsi, sur le plan de la conservativité des volumes, les essais en canal expérimental et en vallées naturelles ont été déterminants : pour obtenir avec la méthode des caractéristiques locales (du premier ordre) des résultats comparables à ceux de la méthode de Lax-Wendroff (du second ordre), il était impératif de choisir un pas spatial $\Delta x$ au moins 5 fois plus petit.

Pour ce qui est des oscillations d'origine numérique, on n'en observa pas avec la méthode des caractéristiques locales qui possède une diffusion numérique d'autant plus importante que le pas spatial est grand. Avec la méthode de Lax-Wendroff, il en apparaissait d'inadmissibles dans les zones d'écoulement très varié. Elles sont la contrepartie de la précision de la méthode, qui propage le long des caractéristiques une valeur interpolée paraboliquement. Il était donc indispensable d'atténuer fortement ces oscillations. Cela fut obtenu par l'introduction de deux processus diffusifs, d'une part une perte de charge singulière lorsque la vitesse diminue fortement entre deux sections de calcul successives, d'autre part une $\theta$-pondération, qui sera décrite au chapitre 3 cidessous. Ce faisant, la conservativité des volumes fut également bien améliorée, puisque couramment obtenue à moins de $2 \%$ près.

Enfin, en ce qui concerne l'accord entre résultats calculés et expérimentaux, il est résumé dans le tableau suivant, relatif aux essais en canal.

\begin{tabular}{|c|c|c|c|c|c|}
\hline \multicolumn{2}{|c|}{$\begin{array}{c}\text { Ecarts des calculs par rapport } \\
\text { aux essais ( } \%)\end{array}$} & \multirow{2}{*}{$\begin{array}{c}\text { Caracteristiques } \\
\text { locaies } \\
3 x=0.50 \mathrm{~m} \\
-30\end{array}$} & \multirow{2}{*}{\begin{tabular}{|c} 
Caracteristiques \\
locales \\
$\Delta x=0.10 \mathrm{~m}$
\end{tabular}} & \multirow{2}{*}{$\begin{array}{c}\begin{array}{c}\text { Lax. Wendroff } \\
\text { pur } \\
1 \times=0,50 \mathrm{~m}\end{array} \\
-15\end{array}$} & \multirow{2}{*}{$\begin{array}{c}\text { Lax. Wendrolt } \\
\begin{array}{c}\text { pondere } \\
3 x\end{array}=0.50 \mathrm{~m} \\
-10\end{array}$} \\
\hline Celierite du & Fond sec & & & & \\
\hline & Fond mouille & -10 & -5 & -5 & -5 \\
\hline \multirow{2}{*}{$\begin{array}{c}\text { Cotes } \\
\text { maximales }\end{array}$} & Fond sec & 10 & 10 & 10 & 8 \\
\hline & Fond moullie & -10 & $\neq 5$ & \pm 5 & $=5$ \\
\hline
\end{tabular}

Comme on le verra plus loin, la célérité du front d'onde est liée directement à la vitesse liquide dans la partie aval du corps de l'onde. Cette célérité est donc représentative des vitesses calculées pour l'onde. A remarquer par ailleurs que, s'agissant de méthodes explicites de calcul, une diminution du pas spatial $\Delta x$ entraîne une diminution du pas temporel $\Delta t$, lié au premier par la condition de Courant-Friedrich-Lévy (CFL).

A nouveau, ces comparaisons étaient en faveur de la méthode de Lax-Wendroff (pondéré) qui s'avérait plus sûre, et dont le bilan est positif, même en tenant compte de temps de calcul de 40 à $50 \%$ plus élevés avec le même pas spatial.

\section{Principales caractéristiques du code de calcul du LNH}

Ainsi définies les bases du système d'étude, il restait à structurer le code de calcul dont la vocation serait la prise en charge de l'onde de submersion depuis l'instant de la rupture du barrage. Au cours de la propagation de cette onde, certains phénomènes sont prédéterminés, d'autres dépendent des conditions locales. Les différents traitements particuliers sont décrits ci-après.

\subsection{Initialisation de l'onde}

L'effacement d'un barrage peut être plus ou moins brusque. C'est essentiellement la nature du barrage qui conditionne l'allure de sa rupture (potentielle), plus encore que les causes de cette rupture. C'est ainsi qu'un barrage en béton est susceptible de se rompre quasiinstantanément, comme soufflé par une véritable explosion. La ruine d'une digue en remblais naturels ne saurait au contraire être instantanée, car elle est due à des phénomènes d'érosion. On voit à titre d'exemple à la figure 3 l'évolution du renard ayant abouti à la ruine du barrage Teton (USA).

Dans un souci à la fois de sécurité et de réalisme, on a distingué pour l'hypothèse de rupture entre barrages en béton et digues en remblais. Pour les premiers, c'est la rupture totale et instantanée qui a été retenue par le C.T.P.B. dans la grande majorité des cas, alors que pour les seconds on a envisagé différents processus de rupture progressive par érosion.

* Rupture totale et instantanée : Pour tous les barrages en béton (voûtes, poids-voûtes, poids, à contreforts, etc...) on considère donc cette hypothèse extrême. Les solutions existantes pour initialiser le calcul de l'écoulement consécutif à un tel effacement du barrage sont déduites des équations de Saint-Venant et des équations de l'onde de choc.

Deux voies sont ouvertes dans l'analyse du phénomène, selon que l'on considère ou non les frottements comme négligeables. Une étude expérimentale a montré toutefois que les écarts entre les deux solutions sont faibles, aucune des deux ne devant du reste être appliquée au-delà d'un court laps de temps après l'instant de la rupture. La solution sans frottement étant la plus simple et allant dans le sens de la sécurité, a été adoptée. Il faut alors distinguer si l'aval immédiat du barrage est sec ou s'il y existe initialement une étendue d'eau de profondeur non négligeable.

Dans le premier cas, les équations de Saint-Venant tronquées du terme de frottement admettent des droites centrées sur le barrage comme courbes caractéristiques (courbes le long desquelles les invariants de Riemann sont constants). La prise en compte de courbes analytiques simples (rectangle, parabole ou triangle) pour exprimer de façon approchée le profil de la vallée au barrage conduit à des expressions bien connues du débit et des tirants d'eau sur le front d'onde naissant. Rappelons que cette solution a été imaginée par Ritter, et décrite dans un mémoire de 1892 [5].

S'il existe à l'aval du barrage des fonds mouillés, le profil du front d'onde - décrit par Stoker en 1957 [6] - 


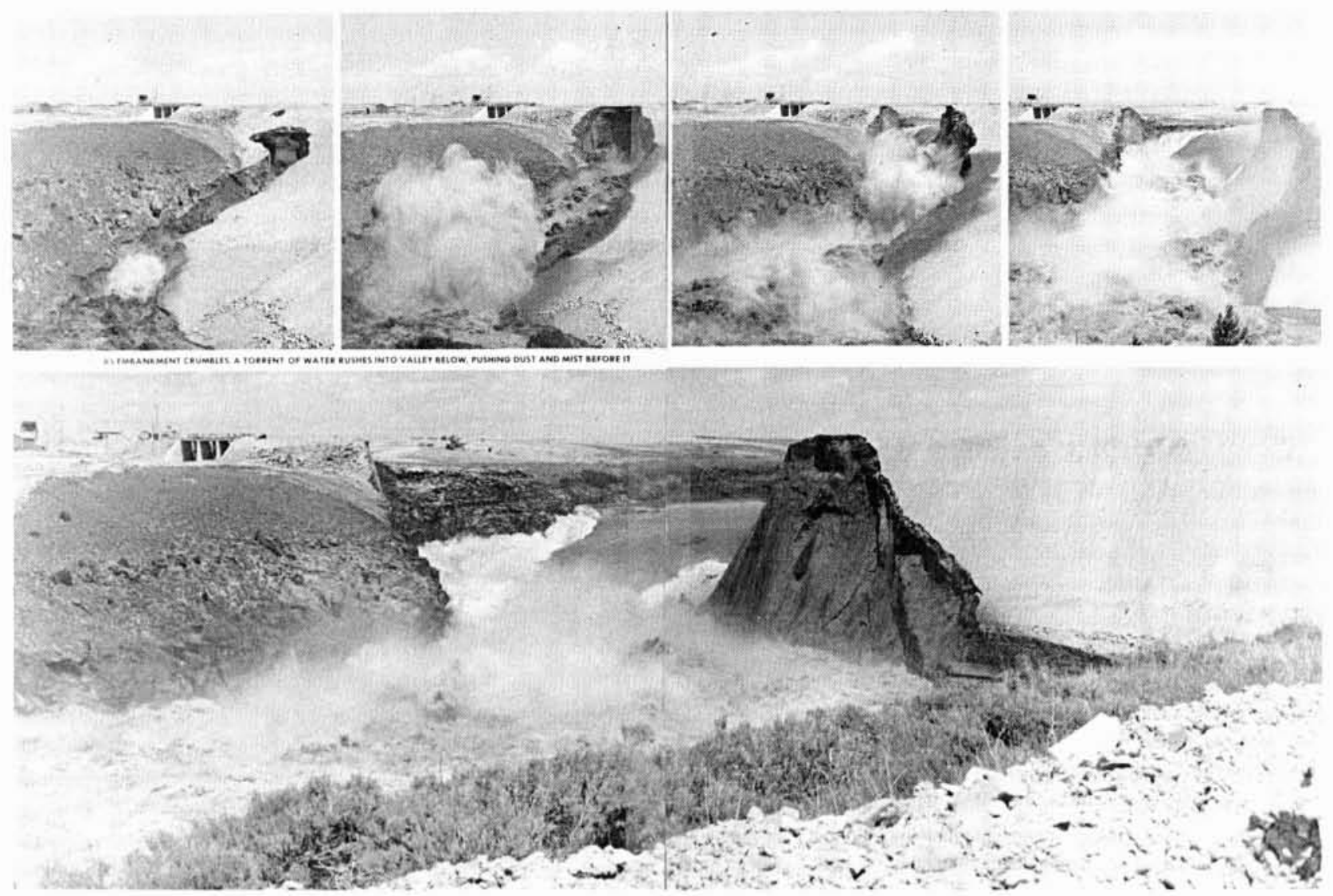

3. Phases successives de la rupture du barrage Teton.

s'obtient en superposant à la solution de Ritter une onde de choc derrière laquelle s'étend un domaine de vitesse et de hauteur d'eau constantes.

* Rupture progressive: Les accidents recensés à ce jour font état pour les digues en remblai de deux modes de destruction, soit par suite d'un renard (c'est-à-dire une fuite par érosion interne) apparu dans le corps de l'ouvrage, soit par surverse sur tout ou partie de la crête due à une crue ou à l'onde de rupture d'un barrage amont. Ces deux circonstances ont été modélisées ([7] et [8]), et les méthodes de calcul testées à partir de mesures sur modèle réduit et de relations de témoins de ruptures réelles. Les processus d'érosion sont évidemment schématisés à partir des caractéristiques moyennes des matériaux constitutifs de la digue. On a cherché avant tout un accord satisfaisant avec l'observation pour ce qui concerne les dimensions finales de la brèche et le temps d'obtention de celle-ci, qui sont les paramètres prépondérants dans les caractéristiques de l'onde de submersion.

La modélisation de l'évolution du renard est basée sur les lois de charriage de Meyer-Peter. Il est de plus supposé que pour un certain diamètre du renard, la voûte le surplombant s'effondre brutalement pour donner naissance à la brèche proprement dite, ce qui est conforme à l'observation.

La simulation de l'érosion par surverse fait appel au calcul de la ligne d'eau à prédominance torrentielle sur le parement aval de la digue. Les vitesses liquides considé- rables rencontrées alors ont nécessité d'ajuster les lois de Meyer-Peter, établies en écoulements fluviaux. Des essais d'érosion d'une digue réduite en canal expérimental ont ainsi pu être simulés par le calcul (voir fig. 4), ainsi que la rupture du barrage Oros, au Brésil.

A noter que dans tous les cas de rupture ainsi calculés, la loi de débit au pied du barrage est beaucoup moins brutale qu'en cas de rupture totale et instantanée.

\subsection{Propagation de l'onde dans la vallée}

L'onde une fois formée poursuit sa course dans la vallée. Ses caractéristiques évoluent en fonction de la topographie rencontrée. Cela justifie la prise en compte d'une modélisation de la vallée la plus fidèle possible : ce point sera détaillé plus loin. Encore fallait-il trouver une méthode de calcul des paramètres de l'onde capable de supporter - sauf de rares exceptions exposées par ailleurs - les accidents d'une vallée naturelle. C'est dans ce but qu'a été choisie la méthode particulière de résolution des équations de Saint-Venant du code de calcul du LNH à la suite des tests décrits au chapitre précédent. Par ailleurs, les hypothèses de Saint-Venant tombent, comme on le sait, en défaut sur les chocs, phénomènes fréquents au sein des ondes de submersion. Ainsi se justifie la solution adoptée à l'époque de la mise au point du code, consistant en un traitement particulier de ces chocs (shock fitting). 

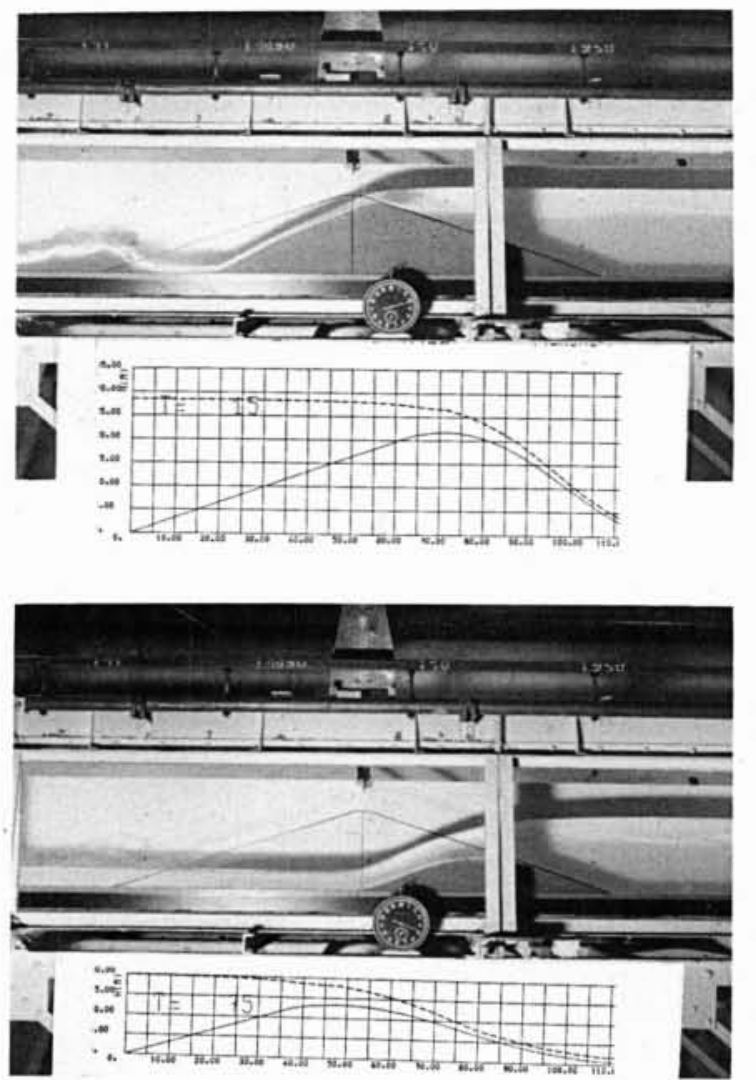

4. Simulation de l'érosion par surverse d'une digue expérimentale. Phases successives, observées et calculées, de l'érosion (NB : les diagrammes sont inversés par rapport aux vues).
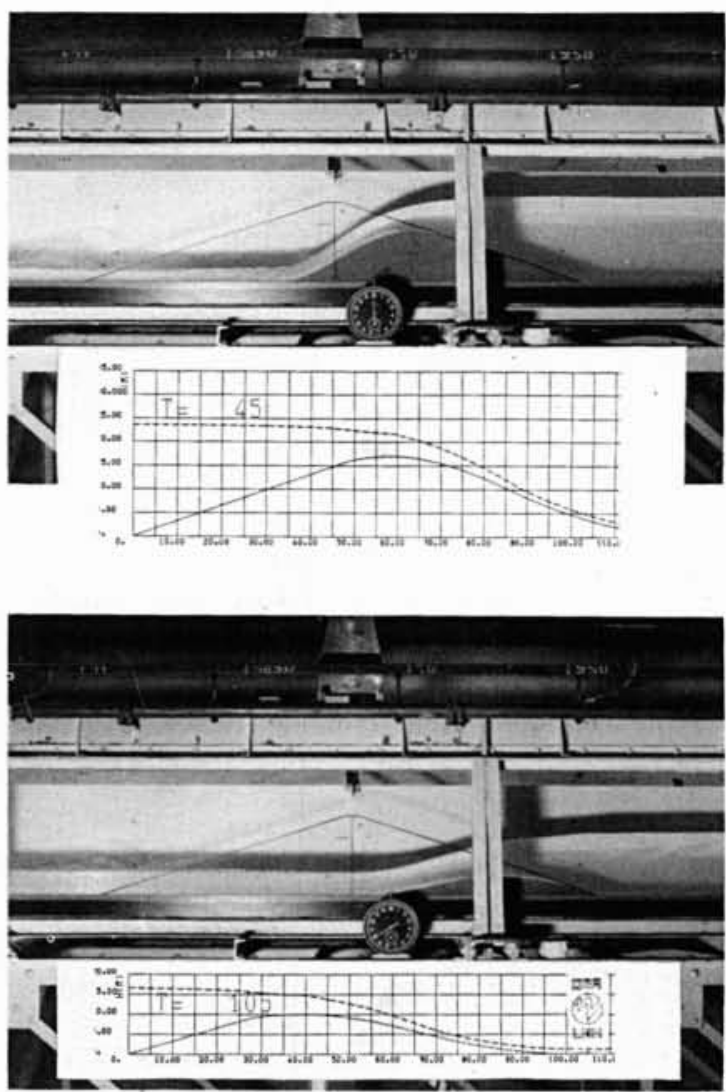

Remarque: Il s'agissait là d'un choix délibéré. En effet, il s'avère que les équations de Saint-Venant étendues au sens des distributions - donnent les mêmes résultats que les relations du choc, car elles traduisent les mêmes bilans de masse et de quantité de mouvement. De là certaines méthodes récentes dans lesquelles le traitement des chocs n'est pas dissocié du calcul des points ordinaires de l'onde (shock capturing). Au passif de telles méthodes, on peut cependant indiquer la nécessité d'introduction dans le schéma de résolution d'une atténuation artificielle locale des oscillations parasites.

* Méthode de résolution: Les hypothèses de base des équations de Saint-Venant monodimensionnelles stipulent que les vitesses ponctuelles sont voisines et de même direction que la vitesse. moyenne dans une section d'écoulement, et que le gradient des pressions est hydrostatique. La forme donnée au terme source de perte de charge linéaire suppose par ailleurs que celle-ci est équivalente à celle de l'écoulement uniforme tangent, c'est-à-dire qui écoule le même débit dans la même section mouillée.

Les équations de Saint-Venant sont écrites sous une forme conservative (sans coefficient à l'extérieur des dérivées) :

$$
\begin{array}{ll}
\text { équation de continuité } & \frac{\partial S}{\partial t}+\frac{\partial Q}{\partial x}=0 \\
\text { équation dynamique } & \frac{\partial V}{\partial t}+\frac{\partial E}{\partial x}=F
\end{array}
$$

avec $E=V^{2} / 2+g Z, F=-g(J+\xi)$ en utilisant les notations usuelles : $g=$ accélération de la pesanteur, $x=$ abscisse curviligne le long de la vallée, $t=$ temps, $S=$ section, $Q=$ débit, $V=$ vitesse moyenne, $Z=$ cote de la surface libre, $J=$ pente de la ligne d'énergie, $\xi=$ perte de charge singulière. La première particularité de la méthode apparaît dans la formulation de l'équation de continuité en variables hauteur-débit, condition nécessaire pour assurer de façon rigoureuse la conservativité de la masse à travers la discrétisation. Le schéma de discrétisation adopté est calqué sur le schéma de LaxWendroff, auquel est adjoint une pondération dans la discrétisation en temps du terme de frottement.

Cette procédure a été adoptée pour ses capacités à propager les ondes avec des amortissements et des déphasages minimaux. Ce schéma essentiellement explicite est appliqué en respectant le critère de Courant (CFL) maximal de l'écoulement instantané, ce qui oblige à recalculer le pas temporel à chaque étape de calcul. Cette méthode, qui peut être jugée lourde et coûteuse en 
Lextrémité du canal d'essais qui représente un barrage aval heurté par conde de submersion incidente, est pripé de captenus de mesures pression sur toute sa hauteur.
Lors de limpact de l'onde, la pression exercée sur la partie initialement l"évolution de la hauteur de leau (effet statique).
Dans le cas où l'onde incidente est deferlante, on observe sur la partie du barrage initialement a lair libre (poin B), un pic de pression très bref dâ a leffet dymamique qui survient avant effet statique.

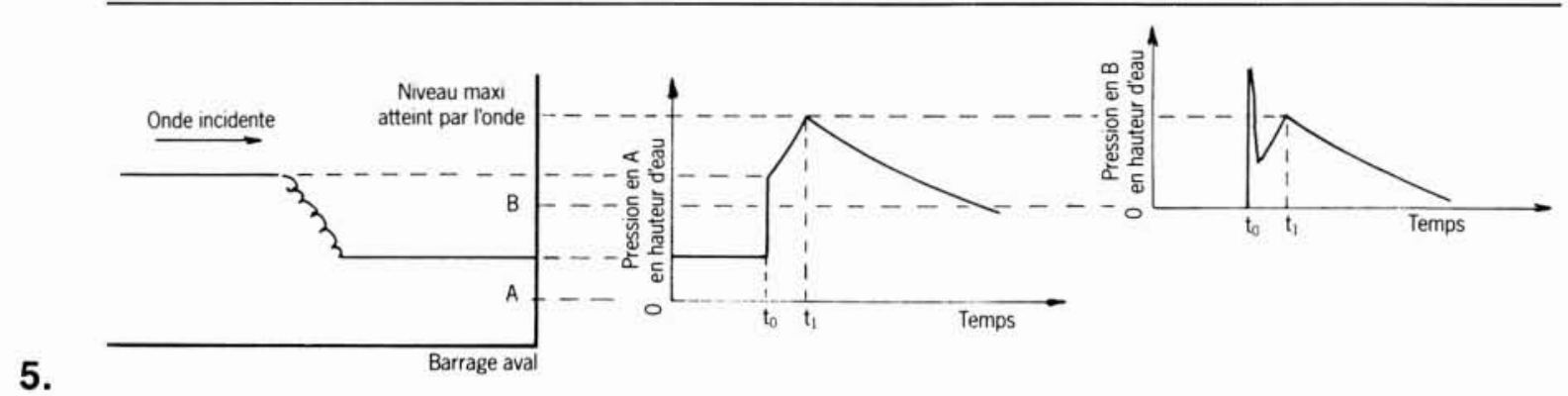

temps-calcul, a été trouvée la mieux adaptée aux forts gradients couramment rencontrés au sein des ondes de submersion. Un développement détaillé du schéma de calcul est fourni en annexe.

* Traitement des singularités : La délimitation et le calcul par le code des différents tronçons du corps de l'onde où l'écoulement est graduellement varié (points courants) une fois effectués, il reste à calculer l'écoulement aux différentes singularités. Celles-ci peuvent être mobiles, comme le front d'onde et les ressauts, ou fixes comme les barrages aval et les confluents éventuels.

* Le front d'onde: Lorsque la propagation a lieu dans une vallée initialement sèche, l'expérience a montré qu'il existe en avant de l'onde un bourrelet d'eau au sein duquel les vitesses liquides sont pratiquement constantes - abstraction faite de l'agitation turbulente apparaissant au pied de ce bourrelet - et constituent la célérité du front d'onde.

Ce résultat expérimental a été utilisé dans le code pour calculer la progression du front d'onde. Pour cela, la zone du front est dissociée du corps de l'onde calculé à partir du système d'équations de Saint-Venant. La dernière vitesse (aval) ainsi calculée est prise comme vitesse constante dans le front, et célérité du point limite à tirant d'eau nul. Le problème est fermé par la prise en compte de l'équation de continuité à l'intérieur du front d'onde.

Lorsque l'onde se propage sur un tirant d'eau initial, comme dans le cas d'une crue importante ou d'une retenue, il se forme le plus souvent un mascaret en avant de l'onde. Celui-ci est traité comme un choc: à travers l'onde de choc, la pression n'est plus hydrostatique et l'accélération verticale n'est pas négligeable. Il faut détecter l'apparition - et la disparition - d'un tel phénomène, et calculer ses caractéristiques. La détection repose sur la propriété de l'onde de choc d'être plus rapide que les intumescences se propageant sur le tirant d'eau aval, mais d'être rattrapée par celles se propageant sur le tirant d'eau amont. Le calcul du choc exploite ces mêmes propriétés, qui permettent d'utiliser les relations de cotes et débits sur une caractéristique d'amont et deux caractéristiques d'aval se joignant sur la courbe de propagation de l'onde de choc. L'équation de quantité de mouvement ferme le problème, et permet de calculer les paramètres amont et aval ( 2 cotes et 2 débits) de l'onde de choc ainsi que sa célérité. C'est donc une formulation du premier ordre.

* Les ressauts: La théorie de l'onde de choc s'applique également dès qu'un ralentissement de l'écoulement provoque un changement de régime, qui passe du torrentiel à l'amont au fluvial à l'aval, ce qui correspond physiquement à l'apparition d'un ressaut. Du point de vue théorique, un ressaut est une onde de choc. La méthode adoptée (shock fitting) consiste à détecter les changements de régime au sein de l'onde, à découpler les zones correspondantes du reste de l'écoulement, et à y appliquer le même traitement que pour le mascaret (en intervertissant les zones amont et aval).

* Les barrages aval: Dans le cas où existent plusieurs barrages sur la même vallée, les ouvrages aval sont concernés par la rupture d'un barrage amont. Leur comportement sous l'effet de l'onde amont est une hypothèse de calcul qui doit être prise avec soin. La règle adoptée en France consiste en général à supposer que les barrages de types poids, à voûtes multiples ou à contreforts, et les barrages mobiles s'effacent totalement et instantanément lorsqu'ils sont atteints par l'onde amont. Au contraire, on admet que les barrages-voûtes résistent au flot et se comportent en déversoirs. Naturellement, cette règle supporte des exceptions dans des cas particuliers. Pour aider au choix de l'hypothèse la plus réaliste, le $\mathrm{LNH}$ a étudié sur modèle réduit la répartition et l'intensité des contraintes exercées par une onde incidente sur un barrage, selon leurs caractéristiques réciproques [9].

Les essais ont montré que, selon les conditions initiales, la forme de l'onde de rupture est très variable et peut prendre l'aspect d'un écoulement proche de la houle ou d'une vague déferlante. Dans le premier cas la variation de pression à l'arrivée de l'onde de choc sur le barrage a l'allure représentée à la figure $5 a$; dans le second, on observe un pic de pression dû à un effet dynamique, comme représenté à la figure $5 b$.

Cela a conduit à introduire dans le calcul des ondes de submersion plusieurs degrés de liberté permettant au code d'auto-régler un certain déphasage entre l'arrivée 
de l'onde et la rupture du barrage touché.

La rupture d'un barrage appartenant à une chaîne est calculée à partir des mêmes conventions que pour l'ouvrage amont, avec pour paramètre supplémentaire la vitesse de l'écoulement amont. Le déversement sur un ouvrage en régime instationnaire est assez délicat. Il nécessite de calculer à chaque instant les conditions d'écoulement sur le déversoir, où s'établit le régime critique correspondant à la charge amont. Celle-ci s'exprimant en fonction du débit critique, le problème conduit à une équation implicite qui ne peut être résolue que par itérations. Mais le point le plus délicat est sans doute la description de l'écoulement au pied du barrage déversant. Le problème n'admet pas de solution rigoureuse, puisque la nappe déversante chute de la hauteur du barrage avec une forte turbulence. Le problème est encore compliqué par l'instationnarité du débit déversé. L'option choisie a consisté à supposer au pied du barrage l'apparition d'un ressaut, dont on calcule l'écoulement aval (en régime fluvial) de façon schématique. Ces conditions permettent de poursuivre le calcul vers l'aval.

* Les confluents: Lorsqu'elle arrive à un confluent, l'onde de submersion se partage entre les deux vallées. La zone du confluent est alors le siège d'un écoulement à deux dimensions privilégiées, et en tant que telle, ne saurait être en toute rigueur traitée dans un modèle filaire. Néanmoins, il a paru hors de propos d'appliquer un traitement bidimensionnel à ces zones, le plus souvent d'étendue restreinte par rapport au modèle d'ensemble. Cette attitude n'interdit du reste pas la mise en œuvre d'un modèle de détail bidimensionnel au cas où la zone du confluent le justifierait.

Les premiers essais de calcul filaire non permanent appliqué aux confluents étaient basés sur la méthode des caractéristiques. Le confluent était représenté par un point singulier triple, les trois branches y aboutissant étant soumises aux relations de continuité. Il s'est rapidement avéré que cette méthode tombait souvent en défaut en présence d'une onde de submersion, où l'écoulement peut être torrentiel dans l'une ou l'autre des branches aval, ce qui annihile une relation sur la caractéristique de la branche correspondante. Après plusieurs essais de schémas pseudo-bidimensionnels, on s'est arrêté à une méthode classique basée sur les équations filaires de Saint-Venant avec un terme de débit d'apport (positif ou négatif) dans l'équation de continuité. La schématisation de l'écoulement suppose de plus la prédominance de l'une des vallées aval sur l'autre. Cela constitue un fait d'observation, soit qu'elle soit la plus large, soit qu'elle ait la même direction que la vallée amont, soit également qu'elle soit de pente descendante, alors que l'affluent est pris à contre-courant par l'onde. La vallée principale étant choisie, on y définit une zone d'échange au sein de laquelle se superposent l'écoulement principal - longitudinal - et l'écoulement secondaire vers (ou en provenance de) l'affluent (voir fig. 6). Le premier de ces écoulements concerne une section mouillée (A) amputée en direction de l'affluent de la partie supposée appartenir à ce dernier. Le second intéresse la section transversale (B) située à la frontière résultant du découpage précédent. Les vitesses $V_{A}$ et $V_{B}$ de ces deux écoulements peuvent être choisies différentes ou égales selon que l'on impose ou non une perte de charge singulière (par changement de direction) à l'écoulement vers l'affluent. On obtient ainsi les débits injectés dans l'affluent, par l'intermédiaire de la section fictive (B) destinée à intégrer de façon simple les cotes calculées dans la zone d'échange et les débits transversaux correspondants.

Cette schématisation donne des résultats satisfaisants lorsque les caractéristiques géométriques des vallées (pentes et sections mouillées) sont continues au confluent, et que l'ampleur du domaine submergé ne varie pas trop entre le début de l'écoulement et son maximum, ce qui est convenablement respecté dans la plupart des ondes raides consécutives à une rupture instantanée de barrage.

\section{Cas particuliers}

Le code de calcul «standard" peut être mis en défaut dans certains cas, qui tiennent à la configuration de la vallée, et entraîneraient manifestement des impossibilités de calcul ou des résultats imparfaits.

Il est alors fait appel soit à un modèle physique, soit à une modélisation mathématique à deux dimensions en plan.

* Modèle physique: Lorsque la vallée à proximité du barrage est par trop accidentée, on a recours à un modèle physique. De telles études ont été effectuées pour des barrages de montagne, avec des vallées de pente raide, de fortes ruptures de pente pouvant donner lieu à des cascades et des sinuosités très accentuées (on verra à la figure 7 le modèle du barrage de Roselend). Les modèles représentaient des tronçons de vallée de quelques kilomètres de longueur, avec des dénivelées pouvant atteindre $1000 \mathrm{~m}$. L'échelle géométrique était le plus souvent le $1 / 500$ sans distorsion, ce qui permettait de conserver le même régime d'écoulement turbulent sur le modèle et en nature. A cette échelle, les fortes rugosités rencontrées dans les torrents de montagne sont traduites assez fidèlement sur un modèle réalisé en ciment lissé ou en résine polyester stratifiée et moulée. La rupture quasi-instantanée du barrage était simulée par un système pneumatique permettant l'effacement (vers le haut ou le bas) en un temps inférieur à la seconde sur le modèle. La propagation de l'onde était enregistrée à l'aide de capteurs de niveaux répartis sur le modèle, et l'enveloppe des cotes maximales atteintes par l'onde repérée sur les parois. La variation temporelle des volumes d'eau à l'extrémité du modèle était obtenue par pesée ; on en déduisait l'hydrogramme à injecter dans le modèle mathématique prenant le relais du modèle physique vers l'aval.

* Modèle bidimensionnel : Le second cas où le modèle filaire est insuffisant est celui où l'onde débouche dans une zone très plate. Quatre barrages français se trouvaient dans ce cas. Les vitesses de l'écoulement n'ont pas alors de direction en plan privilégiée. On peut valablement admettre que leur composante verticale est faible devant les composantes horizontales, et supposer que les forces verticales de viscosité turbulente sont négligeables devant les forces de pesanteur. L'intégration sur la verticale des équations générales de la mécanique des 


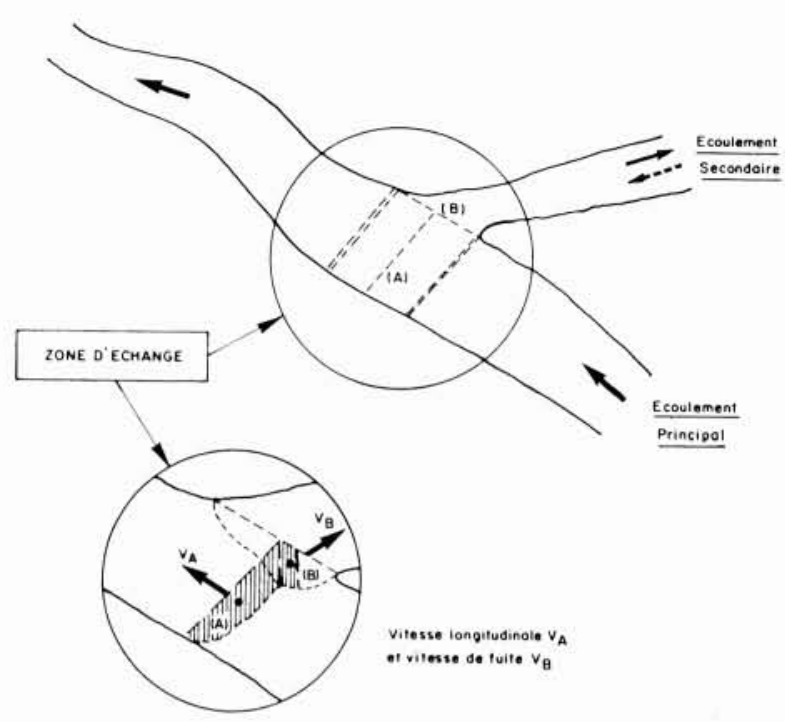

6. Modélisation d'un confluent.

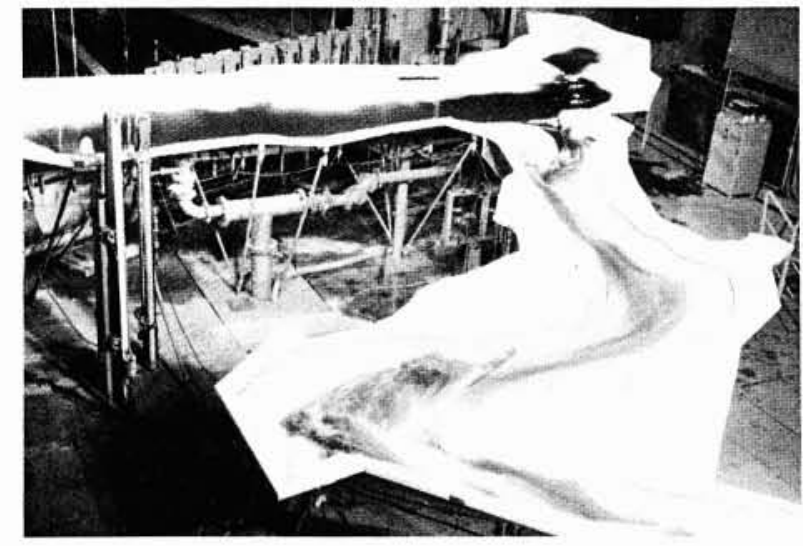

7. La portion de vallée très accidentée à l'aval du barrage de Roselend a nécessité la mise en auvre d'un modèle physique pour étudier l'onde de submersion.

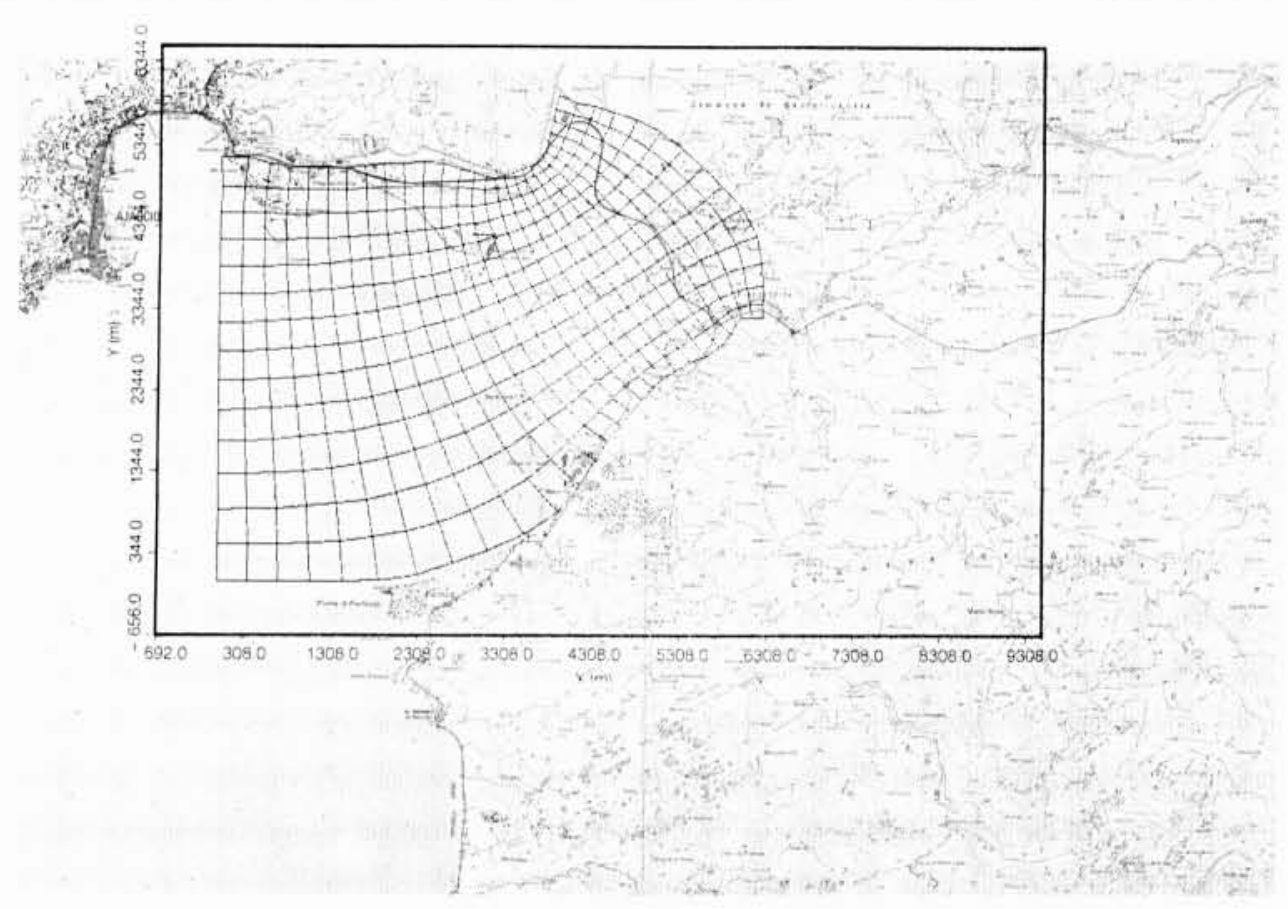

8. Modèle bidimensionnel de la baie d'Ajaccio, avec son maillage.

fluides conduit à un système bidimensionnel en plan. Comme pour le code de calcul filaire, il y a été ajouté une loi de propagation de la zone frontale sur fond sec, similaire à la loi à une dimension. Le code de calcul bidimensionnel est basé sur une méthode de résolution aux différences finies à pas fractionnaires. Cette résolution est réalisée en trois étapes, correspondant aux phénomènes physiques traduits par les équations: convection, diffusion et propagation. La topographie de la zone à étudier est représentée aux nœuds d'un maillage, de mailles assez fines pour représenter le maximum des accidents de relief. A titre d'exemple, on a représenté à la figure 8 la zone littorale (baie d'Ajaccio) reproduite sur modèle bidimensionnel pour l'étude de l'onde de submersion du barrage corse de Tolla, sur le Prunelli, ainsi que le maillage adopté.

Les calculs sont évidemment plus lourds que ceux d'un modèle filaire, et de plus la conservativité des volumes n'est pas toujours aussi bien respectée.

C'est pourquoi on n'a eu recours à une telle modélisation que dans des cas complexes où l'allure bidimensionnelle de l'écoulement était très marquée et risquait d'infirmer les résultats d'une modélisation filaire manifestement trop schématique. 


\section{La modélisation des vallées}

Par les différents procédés qui viennent d'être décrits, on a cherché à simuler la propagation des ondes de submersion, afin d'en déduire les zones submergées avec les moindres risques d'erreur. C'est dans cet esprit qu'a été choisi et développé l'outil principal constitué par le code de calcul filaire, après de nombreux tests expérimentaux. Cependant, le logiciel a un complément indispensable, constitué par la modélisation de la vallée.

La schématisation de la vallée doit en quelque sorte anticiper les caractéristiques de l'onde : à proximité d'un barrage de $50 \mathrm{~m}$ de hauteur, il est probable que l'onde emporterait tout sur son passage et que ni les ponts, ni les édifices, ni les remblais n'auraient d'influence notable sur les caractéristiques de l'écoulement. Au contraire, en s'éloignant de ce même barrage - ou ce qui revient au même, à proximité d'un barrage de moindre importance - les obstacles artificiels ou naturels deviendraient prépondérants sur la ligne d'eau. Le nombre et le choix des profils en travers de définition de la vallée doivent être déterminés en fonction de ces considérations.

Dans l'optique d'une banalisation des calculs d'ondes de submersion, une série de tests a été récemment effectuée sur l'importance de la densité des données et de la prise en compte des sections singulières, notamment en présence d'une onde de hauteur modeste. On a pu constater que l'abandon d'une singularité locale n'altère que localement les résultats, tandis qu'un allègement systématique du nombre de profils de définition d'un modèle aboutit à des résultats complètement différents de ceux du modèle le plus élaboré. Cela justifie le soin apporté à la modélisation de la vallée, qui s'avère aussi fondamental que la qualité du logiciel.

Le choix des caractéristiques hydrauliques de la vallée, concrétisées par les coefficients de rugosité, est également délicat. Ces coefficients englobent en fait la rugosité des terrains submergés, la rugosité de forme de la vallée, ainsi que les pertes d'énergie locales dues notamment aux coudes brusques, non pris en compte par ailleurs. Le choix de ces coefficients n'est justifiable comme on l'a vu d'aucun étalonnage, et ne peut être effectué qu'après examen détaillé des cartes géographiques et prospection approfondie sur les différents sites. Les valeurs des rugosités (coefficients de Strickler) sont affectées en chaque point d'après un catalogue tenant compte de certains critères fixés en accord avec le C.T.P.B., tels que le type de relief, la nature et la densité de la végétation, la présence d'agglomérations à plus ou moins forte densité de constructions. Les coefficients de Strickler de ce catalogue s'étagent entre 15 et 35 (dans le système d'unités SI). Volontairement sévères, ces valeurs comportent une part d'arbitraire. Aussi fut-il dès l'origine des études cherché à évaluer leur influence sur les caractéristiques de l'onde. Dans ce but, une quinzaine de calculs d'ondes ont été effectués avec des coefficients de Strickler de 20 et 40 (SI) successivement, ce qui représente une fourchette considérable. On a constaté que la célérité du front d'onde croissait en moyenne de $70 \%$, mais que l'influence sur les niveaux maximaux était beaucoup moins nette, puisque négligeable dans $30 \%$ des cas étudiés. Pour un coefficient de 20 , ces cotes sont en général plus fortes, en dehors des retenues où elles sont plus faibles que pour 40 . Les écarts des hauteurs d'eau ont atteint jusqu'à $100 \%$ en particulier sur les retenues. Ils sont en moyenne de $20 \%$ dans les premiers kilomètres à l'aval du barrage, et de $10 \%$ audelà de $X / H$ égal à $0,6, X$ étant la distance au barrage (en $\mathrm{km}$ ) et $H$ la hauteur de celui-ci (en mètres). L'incertitude sur les rugosités a conduit à appliquer aux résultats de calcul certaines corrections forfaitaires allant dans le sens de la sécurité, comme indiqué plus loin.

Quant aux modèles bidimensionnels, ils sont constitués par les cotes du terrain naturel aux nœuds d'un maillage couvrant le domaine à étudier. Ce maillage peut être choisi cartésien ou curviligne orthogonal, avec des dimensions de maille variables. Cette possibilité est très utile pour représenter les accidents de relief significatifs. Elle est cependant limitée par les dimensions prohibitives d'un modèle où l'on chercherait à reproduire des obstacles artificiels linéaires, comme des remblais routiers ou ferroviaires. L'évaluation des coefficients de rugosité sur ce maillage est probablement plus encore empirique que dans le cas du modèle filaire.

\section{Etablissement des plans d'alerte}

L'évacuation préventive des populations menacées par une rupture éventuelle de barrage est une mesure efficace, à condition d'être basée sur des estimations fiables des zones submergées. Pour de multiples raisons, ces estimations sont imparfaites, et il s'est imposé à l'esprit des autorités responsables qu'il était préférable de mettre sur pied des plans d'évacuation bâtis à partir de valeurs sécurisantes, sans exagération inutile. L'exploitation des résultats d'étude est ainsi soumise à des règles de sécurité portant sur une diminution forfaitaire des temps calculés d'arrivée de l'onde, et sur une majoration forfaitaire des niveaux d'eau calculés.

Les valeurs ainsi corrigées servent à l'établissement des cartes des zones submergées sur lesquelles sont basés les plans d'alerte (fig. 9). Naturellement, ces prescriptions générales peuvent être adaptées le cas échéant à chaque site particulier par l'autorité responsable.

Enfin, dans un souci d'allègement des mesures à prendre, les dossiers d'étude servant de base aux plans d'alerte sont établis jusqu'à une zone où les niveaux atteints par l'onde de submersion ne dépassent pas ceux de la plus grande crue naturelle observée. L'attention des autorités de protection civile est néanmoins attirée sur la vigilance qui s'imposerait encore à l'aval de cette zone, car les niveaux et les débits y seraient encore exceptionnels, et la montée de l'onde de submersion plus rapide que celle d'une crue naturelle.

\section{Conclusions}

Les études et travaux engagés par EDF au cours des vingt dernières années, et dont le présent article a donné un bref aperçu, ont permis d'acquérir des méthodes et une expérience très importantes dans le domaine original 


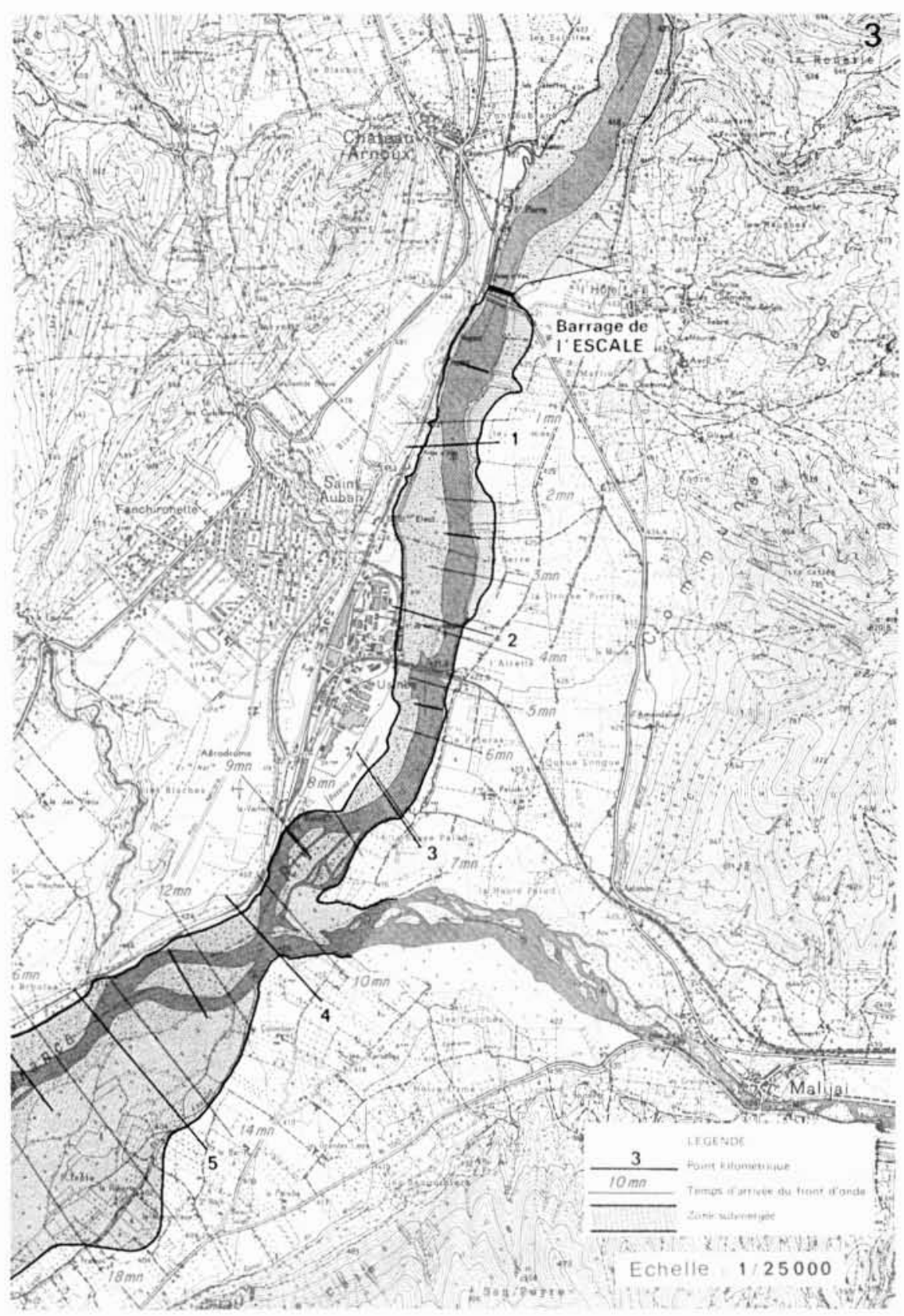

9. Exemple de carte de la zone inondée par l'onde de submersion à l'aval d'un barrage (barrage de l'Escale, sur la Durance).

de l'alerte aux populations menacées par la rupture des barrages. Plus de 80 études ont été réalisées sur des barrages français, et environ une dizaine sur des ouvrages étrangers.

L'instrument de base mis au point est un logiciel de mécanique des fluides, suffisamment testé pour être d'un emploi sûr dans la grande majorité des cas. Une gamme d'outils annexes est disponible pour les cas particuliers de rupture (ruptures progressives par renard ou surverse) ainsi que pour les vallées de conformations délicates (modélisation physique pour les vallées très accidentées, modélisation mathématique bidimensionnelle pour les vallées plates).

Les récents progrès réalisés dans les matériels informatiques permettent d'envisager une adaptation prochaine de l'outil de travail aux micro-ordinateurs.

Il faut toutefois se mettre en garde contre une banalisation excessive des méthodes : comme souvent en hydraulique, la modélisation des vallées est un travail d'ingénieur expérimenté, qui exige beaucoup de soin, sous peine de déboucher sur des résultats aberrants et dangereux. 


\section{Références}

[1] FABRE, L., Etude des ondes de submersion. Essais en canal, Note LNH C44/74-50, 1974.

[2] Onde de crue consécutive à une rupture de barrage, Note LNH HF/0117, 1963.

[3] Etude sur modèle et par calcul de l'écoulement consécutif à la rupture du barrage de Malpasset, Note LNH HF/0116, 1964.

[4] DAUBert, A. et al., Quelques applications de modèles mathématiques à l'étude des écoulements non permanents dans un réseau ramifié de rivières ou de canaux. La Houillle Blanche, $\mathrm{n}^{\circ}$ 7, 1967.

[5] RITTER, A., Die Forpflanzung der Wasserwellen. Zeitschrift des Vereins Deutscher Ingenieure, 1892, Vol. 46.
[6] STOKER, J. J., Water waves, Vol. IV, Chap. 10, Interscience Publishers Inc., New York, 1957.

[7] Benoist, G., Principes du calcul de la rupture progressive d'un barrage par effet de renard, Note LNH E43/79-43, 1979.

[8] Benoist, G., Calcul de l'érosion d'une digue par surverse, Note LNH HE43/83-31, 1983.

[9] SICARD, J. M., Effet d'une onde de rupture sur un barrage aval, Note LNH HE 43/83-43, 1983.

Adresse de l'auteur:

Monsieur G. Benoist, EDF, Laboratoire national d'hydraulique, 6, quai Watier, 78401 Chatou Cedex (France). Tél. : 30.71.78.29

\section{ANNEXE}

\section{Calcul du point courant par un schéma} de Lax-Wendroff avec pondération

Les équations de St-Venant sont écrites sous forme conservative (avec les notations habituelles) :

$$
\begin{aligned}
& \frac{\partial S}{\partial t}+\frac{\partial Q}{\partial x}=0 \\
& \frac{\partial V}{\partial t}+\frac{\partial E}{\partial x}=F
\end{aligned}
$$

où $E=V^{2} / 2+g Z$ et $F=-g(J+\xi)$

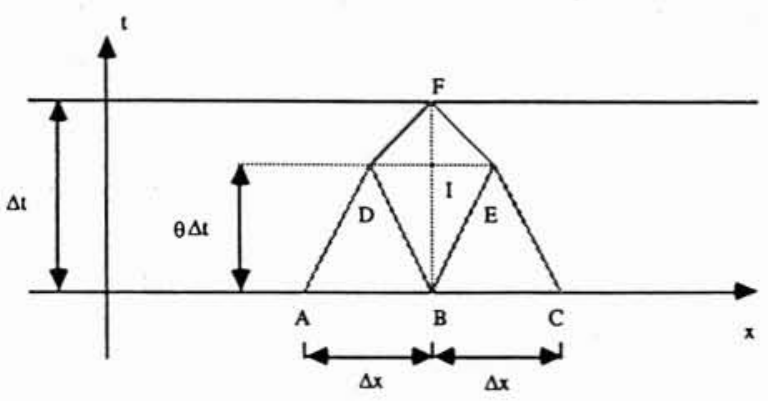

La discrétisation est basée sur le schéma ci-dessus. Dans le triangle $A B D$, on écrit les relations :

$$
\frac{S_{D}-\frac{S_{A}+S_{B}}{2}}{\theta \Delta t}+\frac{Q_{B}-Q_{A}}{\Delta x}=0
$$

et

$$
\frac{V_{D}-\frac{V_{A}+V_{B}}{2}}{\theta \Delta t}+\frac{E_{B}-E_{A}}{\Delta x}=-g\left(\frac{J_{A}+J_{B}}{2}+\xi_{A B}\right)
$$

avec

$$
\begin{gathered}
J=\frac{V|V|}{k^{2} R^{4 / 3}} \text { et } \xi_{A B}=\lambda \frac{V_{A}^{2}-V_{B}^{2}}{2 g} \text { où } \lambda=0 \\
\text { si } V_{A} \leqslant V_{B} \text { et } 0,1 \leqslant \lambda \leqslant 0,3 \text { si } V_{A}>V_{B} .
\end{gathered}
$$

On en déduit :

$$
S_{D}=-\theta \frac{\Delta t}{\Delta x}\left(Q_{B}-Q_{A}\right)+\frac{S_{A}+S_{B}}{2}
$$

et

$$
\begin{aligned}
V_{D}=-g \theta \Delta t\left(\frac{J_{A}+J_{B}}{2}\right. & \left.+\xi_{A B}\right)- \\
& -\theta \frac{\Delta t}{\Delta x}\left(E_{B}-E_{A}\right)+\frac{V_{A}+V_{B}}{2} .
\end{aligned}
$$

On obtient de façon analogue $S_{E}$ et $V_{E}$ dans le triangle $B C E$.

La formulation adoptée au point $I$ résulte des recherches effectuées pour amortir les oscillations parasites. On écrit :

$$
\begin{aligned}
Z_{I} & =\frac{Z_{E}+Z_{D}+\theta Z_{F}+(1-\theta) Z_{B}}{3} \\
\text { et } \quad V_{I}\left|V_{I}\right| & =\left(\theta V_{F}+(1-\theta) V_{B}\right) \frac{\left|V_{E}+V_{D}\right|}{2} .
\end{aligned}
$$

L'équation dynamique (2) discrétisée dans le quadrilatère $B D E F$ aboutit alors à :

$$
\begin{gathered}
V_{F}=V_{B}-\frac{\Delta t}{\Delta x}\left(E_{E}-E_{D}\right)-g \Delta t\left(J_{I}+\xi_{D E}\right) \\
\text { ou } V_{F}=V_{B}-\frac{\Delta t}{\Delta x}\left(E_{E}-E_{D}\right)- \\
-\frac{g \Delta t\left[\theta V_{F}+(1-\theta) V_{B}\right] \frac{\left|V_{E}+V_{D}\right|}{2}}{k_{I}^{2} R_{I}^{4 / 3}}-g \xi_{D E} \Delta t
\end{gathered}
$$

ce qui permet de calculer $V_{F}$.

De même, l'équation de continuité (1) conduit à la cote $Z_{F}$ par l'intermédiaire de la section $S_{F}$ :

$$
S_{F}=S_{B}-\frac{\Delta t}{\Delta x}\left(Q_{E}-Q_{D}\right) .
$$

